05

\title{
Лидарная система комбинационного рассеяния света для зондирования молекул водорода в атмосфере
}

\author{
(C) В.Е. Привалов ${ }^{1}$, В.Г. Шеманин ${ }^{2,3}$ \\ ${ }^{1}$ Санкт-Петербургский политехнический университет Петра Великого, \\ 195251 Санкт-Петербург, Россия \\ ${ }^{2}$ Новороссийский фрилиал Белгородского государственного технологического университета им. В.Г. Шухова, \\ 353919 Новороссийск, Россия \\ ${ }^{3}$ Новороссийский политехнический институт (филиал) Кубанского государственного технологического университета, \\ 353900 Новороссийск, Россия \\ e-mail: vaevpriv@yandex.ru, shemanin-v-g@nb-bstu.ru
}

Поступила в редакцию 03.09.2021 г.

В окончательной редакции 14.11.2021 г.

Принята к публикации 14.11.2021 г.

\begin{abstract}
Выполнено численное решение лидарного уравнения комбинационного рассеяния света для измерения концентрации молекул водорода на уровне $10^{13} \mathrm{~cm}^{-3}$ и выше в атмосфере на расстояниях зондирования до $100 \mathrm{~m}$ в режиме синхронного счета фотонов и сделан выбор оптимальных параметров такого лидара. Показано, что для измерения концентрации молекул водорода $N(z)=10^{13} \mathrm{~cm}^{-3}$ в диапазоне зондирования $z$ от 5 до $100 \mathrm{~m}$ время измерения $t$ лежит в диапазоне от $3.83 \mathrm{~s}$ до $26.5 \mathrm{~min}$, для измерения концентрации $N(z)=10^{15} \mathrm{~cm}^{-3}$ - от $38 \mathrm{~ms}$ до $15.9 \mathrm{~s}$ и для измерения концентрации $N(z)=10^{17} \mathrm{~cm}^{-3}-$ от $400 \mathrm{~ns}$ до $160 \mu \mathrm{s}$.
\end{abstract}

Ключевые слова: лидар комбинационного рассеяния света, молекула водорода, концентрация, расстояние зондирования, длина волны лазерного излучения.

DOI: $10.21883 /$ OS.2022.03.52168.2707-21

\section{Введение}

Большие перспективы использования водорода как нового вида чистого топлива [1] требуют создания систем для контроля его утечки из баллонов и трубопроводов, особенно в местах перекачки. В реальной атмосфере мольная доля молекул $\mathrm{H}_{2}$ составляет $5 \cdot 10^{-7}$ [2] или около $0.5 \mathrm{ppm}$, что соответствует счетной концентрации $1.345 \cdot 10^{13} \mathrm{~cm}^{-3}$. Однако дистанционный контроль и измерение концентраций на таких уровнях представляет большую проблему [3].

Разработка новых лазерных систем и методов измерения концентрации молекул водорода на уровне концентраций порядка $10^{13} \mathrm{~cm}^{-3}$ и выше в атмосфере на базе лидара комбинационного рассеяния света требует выбора оптической схемы и оптимального подбора параметров отдельных элементов такого лидара. Ранее в работе [4] в результате анализа методов лазерного зондирования молекул водорода в атмосфере было установлено, что лидар комбинационного рассеяния света может использоваться для дистанционного измерения концентрации молекул на таком уровне на расстояниях до сотен метров. В работе [5] было предложено лидарное уравнение для комбинационного рассеяния света газовыми молекулами в атмосфере с учетом конечной ширины линии генерации. Оказалось, что учет конечной ширины линии генерации реального лазера, соотношения ширин полос комбинационного рассеяния света и аппаратной функции лидара позволяют уточнить решение такого лидарного уравнения [5].

Целями настоящей работы являются оценка возможности измерений лидаром комбинационного рассеяния света концентрации молекул водорода на уровне $10^{13} \mathrm{~cm}^{-3}$ и выше в атмосфере на расстояниях зондирования до $100 \mathrm{~m}$ в режиме синхронного счета фотонов и выбор оптимальных параметров лидара.

\section{Лидар комбинационного рассеяния света}

Оптическая схема предлагаемого варианта лидара комбинационного рассеяния света биаксиальная, как в $[2,3]$; она приведена на рис. 1 .

В качестве зондирующего излучения используется излучение третьей и второй гармоник $\mathrm{YAG}-\mathrm{Nd}$-лазера (с накачкой полупроводниковым лазером с длинами волн 355 и $532 \mathrm{~nm}$ ) и полупроводникового лазера с длиной волны $405 \mathrm{~nm}$, с длительностью импульсов $10 \mathrm{~ns}$ и энергией в импульсе до $1 \mathrm{~mJ}$ при частоте следования лазерных импульсов $f$ до $100 \mathrm{kHz}$ [2,3]. Излучение лазера 1 направлялось параллельно оси приемного телескопа на расстоянии $150 \mathrm{~mm}$, как и в [2], а излучение комбинационного рассеяния молекулами водорода в атмосфере в направлении назад собиралось приемным телескопом типа Ньютона со сферическим зеркалом 9 диаметром $200 \mathrm{~mm}$ и фокусировалось линзовым объ- 


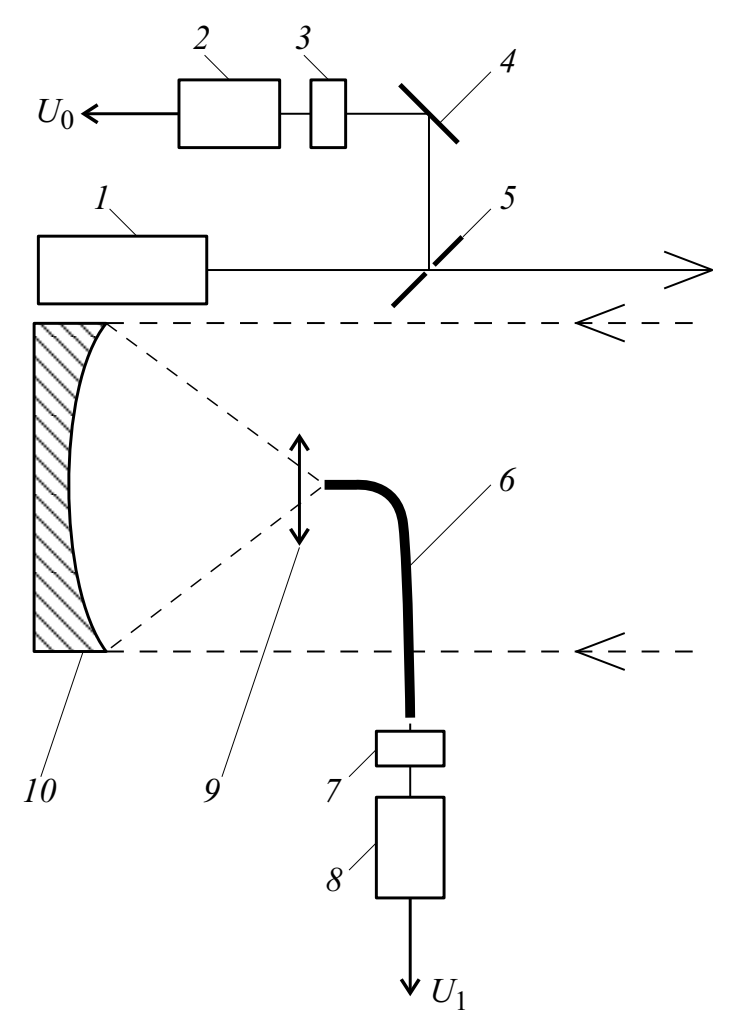

Рис. 1. Оптическая схема лидара комбинационного рассеяния света: 1 - лазер, 2, 8 - фотоприемники, 3, 7 - интерференционные светофильтры, 4 - глухое зеркало, 5 - стеклянная пластина, 6 - волоконный световод, $9-$ линзовый объектив, 10 - сферическое зеркало приемного телескопа.

ективом 8 в волоконный световод 6 и направлялось через интерференционный светофильтр 7 на фотоприемник 8. Выход фотоприемника соединен со входом синхронного детектора, сигнал $U_{1}$ с которого подается на вход аналого-цифрового преобразователя и затем микроконтроллера.

Часть лазерного излучения направлялось стеклянной пластиной 5 и глухим зеркалом 4 через интерференционный светофильтр 3 на фотоприемник 2, напряжение $U_{0}$ с которого записывалось платой сбора данных в ПК для контроля энергии лазерного импульса и формирования начала отсчета времени измерения.

\section{Лидарное уравнение для комбинационного рассеяния света}

Лазер лидара будем характеризовать числом фотонов $n_{0}$ в импульсе посылаемого в атмосферу лазерного излучения длительностью $\tau_{0}$, а линию генерации лазера будем считать гауссовой с максимумом на частоте $v_{0}$ и полушириной $\Gamma_{0}$. Тогда число фотонов $n(v, z)$ излучения комбинационного рассеяния света исследуемыми молекулами водорода с концентрацией $N(z)$ на фотоприемнике лидара можно получить из лидарного уравнения в направлении назад в режиме счета фотонов в виде интеграла в интервале от $\left(v_{0}-\Gamma_{0}\right)$ до $\left(v_{R}-\Gamma_{R}\right)[5]$ :

$$
\begin{aligned}
n(v, z)= & n_{0} c \tau_{1} G(z) f t S_{0} N(z)(d \sigma / d \Omega) \int_{v_{0}-\Gamma_{0}}^{v_{R}+\Gamma_{R}} T_{0}\left(v_{0}, z\right) \\
& \times T\left(v_{R}, z\right) \Phi(v) A(v) d v / 2 z^{2},
\end{aligned}
$$

где $n(v, z)$ - число фотонов, зарегистрированных фотоприемником лидара на частоте $v_{R}$ комбинационного рассеяния света с расстояния зондирования $z, n_{0}$ - число фотонов на частоте $v_{0}$ лазерного излучения. Кроме того, $S_{0}$ - площадь приемной апертуры телескопа, $G(z)-$ геометрический фактор лидара $[2,3]$. Этот фактор определяется параметрами оптической схемы лидара [2,3] и лежит в пределах $0<G(z)<1$. Далее, $N(z)-$ концентрация исследуемых молекул, $t-$ время измерения или накопления сигнала, $(d \sigma / d \Omega)$ - дифференциальное сечение комбинационного рассеяния света исследуемыми молекулами. Два других сомножителя - пропускание атмосферы (как и в $[2,3])$ - равны соответственно

$$
\begin{aligned}
& T_{0}\left(v_{0}, z\right)=\exp \left[-\int_{0}^{z} \alpha\left(v_{0}, r\right) d r\right], \\
& T\left(v_{R}, z\right)=\exp \left[-\int_{0}^{z} \alpha\left(v_{R}, r\right) d r\right],
\end{aligned}
$$

где $\alpha\left(v_{0}, r\right)$ и $\alpha\left(v_{R}, r\right)$ - коэффициенты ослабления на частотах лазерного излучения и комбинационного рассеяния света молекулами водорода:

$$
\begin{aligned}
& n(\nu, z)=n_{0} \frac{c \tau_{0}}{2 z^{2}} G(z) f t S_{0} N(z) \frac{d \sigma}{d \Omega} \\
& \times \int_{v_{0}-\Gamma_{0}}^{v_{R}+\Gamma_{R}} \exp \left[-\int_{0}^{z}\left\{\alpha\left(v_{0}, r\right)+\alpha\left(v_{R}, r\right)\right\} d r\right] \Phi\left(v^{\prime}\right) A\left(v^{\prime}\right) d v^{\prime},
\end{aligned}
$$

где $\Phi(v)$ - функция распределения фотонов лазерного излучения в линии генерации [6]; в нашем случае она гауссова и может быть записана как

$$
\Phi(v)=\frac{1}{\sqrt{2 \pi} \Gamma_{0}} \exp \left[-\frac{\left(v-v_{0}\right)^{2}}{2 \Gamma_{0}^{2}}\right]
$$

Кроме того, будем считать, что аппаратная функция (или спектральный коэффициент пропускания приемной системы лидара $A(v)[2,3,5])$ имеет лоренцеву форму и настроена точно на частоту $v_{R}$ полосы комбинационного рассеяния света молекулами водорода с полушириной $\Gamma_{a}$, которая больше, чем $\Gamma_{R}$ и $\Gamma_{0}$,

$$
A(v)=\frac{K_{1} \xi\left(v_{R}\right)}{\pi} \frac{\Gamma_{a}}{\left(v-v_{R}\right)^{2}+\Gamma_{a}^{2}},
$$

где $K_{1}$ - лидарная константа на частоте $v_{0}$ лазерного излучения, измеренная экспериментально в [3], 
Длины волн и частоты лазерного излучения и полос комбинационного рассеяния света молекулами водорода, дифференциальные сечения колебательного комбинационного рассеяния света (КРС) для этих полос, коэффициенты ослабления в атмосфере на этих длинах волн и относительная спектральная чувствительность фотоприемника

\begin{tabular}{c|c|c|c|c|c}
\hline $\begin{array}{c}\text { Длина волны, } \\
\lambda, \mathrm{nm}\end{array}$ & $\begin{array}{c}\text { Частота, } v, \\
\mathrm{GHz}\end{array}$ & $\begin{array}{c}\text { Дифференциальное } \\
\text { сечение КРС } \\
(d \sigma / d \Omega), 10^{30} \mathrm{~cm}^{2}\end{array}$ & $\begin{array}{c}\text { Коэффициент } \\
\text { ослабления } \\
\alpha\left(\nu_{0}, r\right), \mathrm{km}^{-1}\end{array}$ & $\begin{array}{c}\text { Коэффициент } \\
\text { ослабления } \\
\alpha\left(\nu_{R}, r\right), \mathrm{km}^{-1}\end{array}$ & $\begin{array}{c}\text { Относительная спектральная } \\
\text { чувствительность } \\
\text { фотоприемника } \xi(v)\end{array}$ \\
\hline 355 & 845.1 & 21.7 & 0.31 & & 0.52 \\
\hline 389.3 & 809.5 & & & 0.28 & 0.86 .25 ФЭУ \\
\hline 532 & 563.9 & $4.3 \pm 0.9-21 \%$ & 0.17 & & 0.14 \\
\hline 683.2 & 439.1 & & & & 0.19 \\
\hline 405 & 740.7 & 12.8 & & 0.19 & 0.89
\end{tabular}

а $\xi\left(v_{R}\right)$ - относительная спектральная чувствительность фотоприемника на частоте $v_{R}$ излучения комбинационного рассеяния света. Положим, что $\Phi(v)=1$, когда полуширина линии генерации много меньше, чем полуширина аппаратной функции лидара [5]. Перепишем уравнение (2) в виде

$$
n(v, z)=\frac{B}{z^{2}} N(z) t I(v, z)
$$

в этом выражении введены константа $B$,

$$
B=\frac{c \tau_{1}}{2} S_{0} f
$$

и функция $I(v, z)$,

$$
\begin{aligned}
& I(v, z)=n_{0} G(z) \frac{d \sigma}{d \Omega} \\
& \times \int_{v_{0}-\Gamma_{0}}^{v_{R}+\Gamma_{R}} \exp \left[-\int_{0}^{z}\left\{\alpha\left(v_{0}, r\right)+\alpha\left(v_{R}, r\right)\right\} d r\right] A\left(v^{\prime}\right) d v^{\prime}
\end{aligned}
$$

Далее, полагая коэффициенты ослабления на частотах лазерного излучения и комбинационного рассеяния света молекулами водорода, $\alpha\left(v_{0}, r\right)$ и $\alpha\left(v_{R}, r\right)$, мало зависящими от частоты, перепишем функцию (7) в виде

$$
\begin{aligned}
I(v, z)= & n_{0} G(z) \frac{d \sigma}{d \Omega} \exp \left[-\int_{0}^{z}\left\{\alpha\left(v_{0}, r\right)+\alpha\left(v_{R}, r\right)\right\} d r\right] \\
& \times \int_{v_{0}-\Gamma_{0}}^{v_{R}+\Gamma_{R}} A\left(v^{\prime}\right) d v^{\prime}
\end{aligned}
$$

где последний интеграл

$$
\begin{aligned}
\int_{v_{0}-\Gamma_{0}}^{v_{R}+\Gamma_{R}} A\left(v^{\prime}\right) d v^{\prime} & =\frac{K_{1} \xi\left(v_{R}\right) \Gamma_{a}}{\pi} \int_{v_{0}-\Gamma_{0}}^{v_{R}+\Gamma_{R}} \frac{d v^{\prime}}{\left(v^{\prime}-v_{R}\right)^{2}+\Gamma_{a}^{2}} \\
& =\frac{K_{1} \xi\left(v_{R}\right) \Gamma_{a}}{\pi} \frac{\pi}{2 \Gamma_{a}}=\frac{K_{1} \xi\left(v_{R}\right)}{2} .
\end{aligned}
$$

Тогда выражение (8) приобретает вид

$$
\begin{aligned}
I(\nu, z)= & n_{0} G(z) \frac{d \sigma}{d \Omega} \frac{K_{1} \xi\left(v_{R}\right)}{2} \\
& \times \exp \left[-\int_{0}^{z}\left\{\alpha\left(v_{0}, r\right)+\alpha\left(v_{R}, r\right)\right\} d r\right] .
\end{aligned}
$$

Также полагаем, что дифференциальное сечение комбинационного рассеяния света можно считать постоянным внутри интервала интегрирования по частоте, как и коэффициенты ослабления на частотах лазерного излучения и комбинационного рассеяния света молекулами водорода [7]. Выразим из уравнения (2) время измерения $t$ как меру эффективности лидарной системы и рассмотрим спектральную и концентрационную зависимости решения этого уравнения:

$$
t=\frac{n(v, z) z^{2}}{B I(v, z) N(z)} .
$$

Далее будем рассматривать однократное комбинационное рассеяние света и однородную атмосферу $[2,3,7]$.

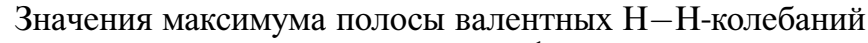
молекул водорода равно $4161 \mathrm{~cm}^{-1}$ по данным [2-4,8], и для всех длин волн лазерного излучения были рассчитаны длины волн полос колебательного комбинационного рассеяния света молекулами водорода; они приведены в таблице.

Значения дифференциальных сечений комбинационного рассеяния света $(d \sigma / d \Omega)$ для всех молекул и выбранных длин волн лазерного излучения $(355,532$ и $405 \mathrm{~nm}$ ) были определены по данным, экспериментально измеренным в [2,8] при комнатной температуре и нормальном давлении на длине волны излучения $532 \mathrm{~nm}$, $(d \sigma / d \Omega)=(4.3 \pm 0.9) \cdot 10^{-30} \mathrm{~cm}^{2} / \mathrm{sr}$, и для интересующих нас длин волн рассчитаны с учетом зависимости $1 / \lambda^{4}$ [3]. Все эти значения приведены в таблице. Значения коэффициентов ослабления для этих частот $\alpha\left(v_{0}, z\right)$ и $\alpha(v, z)$ из [9] также собраны в таблице. 
Поперечное сечение зеркала приемного телескопа лидара было $0.031 \mathrm{~m}^{2}$, а шаг по расстоянию - $7.5 \mathrm{~m}$ для времени одного измерения $50 \mathrm{~ns}$.

\section{Решение лидарного уравнения и обсуждение результатов}

Численно решим уравнение (11), полагая, что $G(z)$ в нашем случае равно 1 и концентрация молекул водорода $N(z)$ лежит в диапазоне $10^{13}-10^{17} \mathrm{~cm}^{-3}$. Для нашей экспериментальной ситуации за время измерения $t$ может быть зарегистрировано 200 фотонов [6].

Для этого случая решим уравнение (11) для трех значений длин волн лазерного излучения (355, 532 и $650 \mathrm{~nm}$ ) при частоте следования лазерных импульсов $100 \mathrm{kHz}$, энергии в импульсе $1 \mathrm{~mJ}$ в диапазоне расстояний зондирования от 5 до $100 \mathrm{~m}$. Результаты решения уравнения (11) для этого случая представлены на рис. 2 в виде зависимости времени измерения $t$ от расстояния зондирования $z$.

На кривых рис. 2 видно увеличение времени измерения $t$ в ряду длин волн лазерного излучения 405-532$355 \mathrm{~nm}$ за счет спектральной зависимости величин, входящих в уравнение (11), при этом время измерения резко возрастает на первых 20 m примерно на порядок.

Далее рассмотрим численное решение уравнения (11) для диапазона концентрации молекул водорода $N(z)$ от $10^{13}$ до $10^{17} \mathrm{~cm}^{-3}$. Для этого решим уравнение (11) для длины волны лазерного излучения $405 \mathrm{~nm}$ как наилучшего варианта по данным рис. 2. Результаты решения уравнения (11) представлены на рис. 3. Время измерения $t$ для нашей экспериментальной ситуации убывает обратно пропорционально концентрации молекул водорода $N(z)$, как и следует из уравнения (11), и растет примерно на два порядка с увеличением расстояния зондирования $z$ от 5 до $100 \mathrm{~m}$.

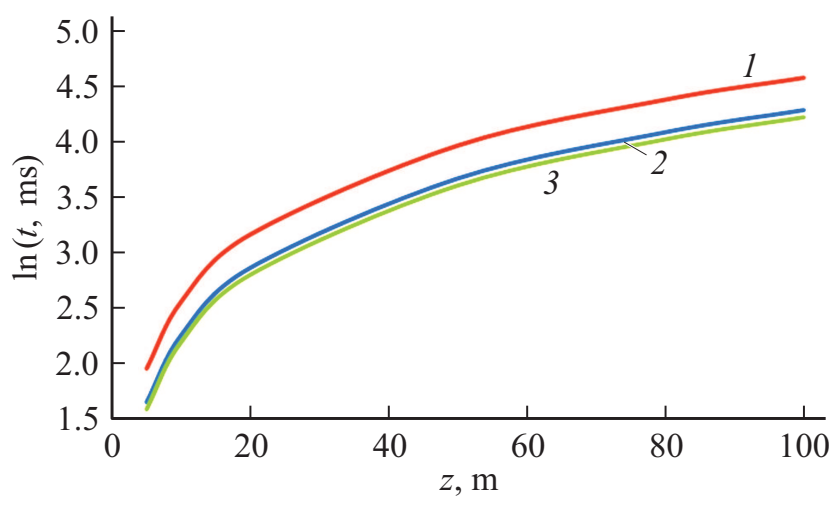

Рис. 2. Кривые рассчитанной по уравнению (11) зависимости времени измерения $t$ для молекул водорода с концентрацией $N(z)=10^{15} \mathrm{~cm}^{-3}$ (в логарифмическом масштабе, $t$ в $\mathrm{ms}$ ) от расстояния зондирования $z$ (в $\mathrm{m}$ ) для длин волн лазерного излучения 355 (1), 532 (2) и $405 \mathrm{~nm}$ (3) при зондировании атмосферы лидаром комбинационного рассеяния света.

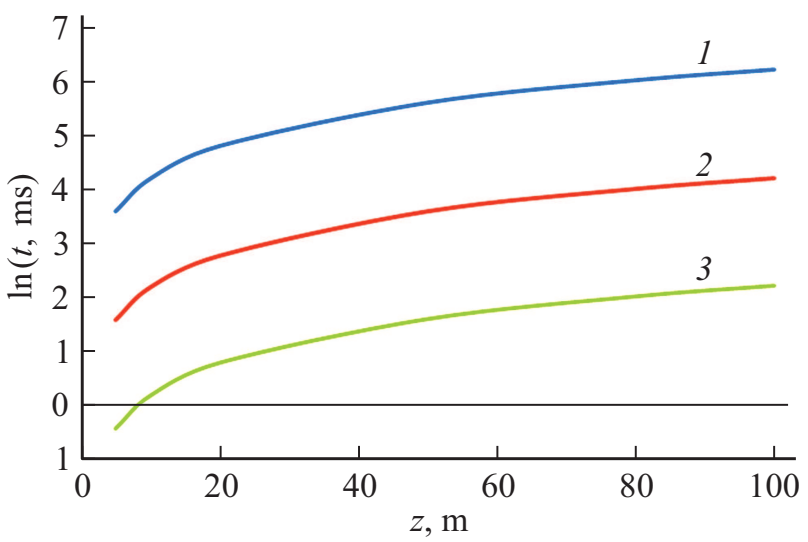

Рис. 3. Кривые рассчитанной по уравнению (11) зависимости времени измерения $t$ (в логарифмическом масштабе, $t$ в $\mathrm{ms}$ ) для молекул водорода при их концентрациях $N(z) 10^{13}(1)$, $10^{15}(2)$ и $10^{17} \mathrm{~cm}^{-3}$ (3) от расстояния зондирования $z$ (в $\left.\mathrm{m}\right)$ для длины волны лазерного излучения $405 \mathrm{~nm}$ для той же экспериментальной ситуации.

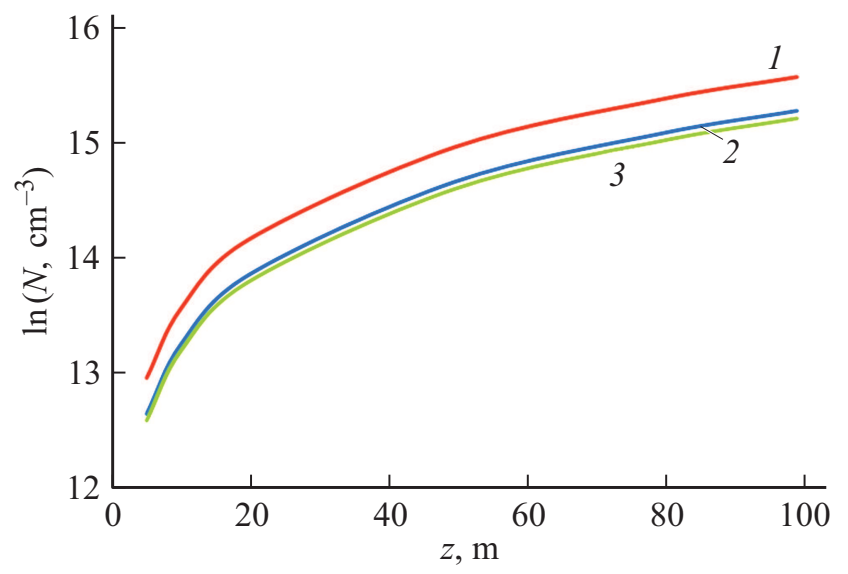

Рис. 4. Кривые рассчитанной по уравнению (12) зависимости концентрации молекул водорода $N(z)$ (в логарифмическом масштабе, в $\mathrm{cm}^{-3}$ ) от расстояния зондирования $z$ (в $\left.\mathrm{m}\right)$ для одного и того же времени измерения $t=10 \mathrm{~s}$ для длин волн лазерного излучения 355 (1), 532 (2) и $405 \mathrm{~nm}$ (3) для той же экспериментальной ситуации.

Более наглядно зависимость концентрации молекул водорода $N(z)$ от расстояния зондирования $z$ для одного и того же времени измерения $t=10 \mathrm{~s}$ для всех длин волн лазерного излучения можно представить, выразив из уравнения (11) величину $N(z)$ :

$$
N(z)=\frac{n(v, z) z^{2}}{B I(v, z) t} .
$$

Далее выполним численное решение уравнения (12) для концентраций молекул водорода $N(z)$ от $10^{13}$ до $10^{17} \mathrm{~cm}^{-3}$, времени измерения $10 \mathrm{~s}$ и всех длин волн лазерного излучения. Результаты решения уравнения (12) представлены на рис. 4. 
Кривые на рис. 4 показывают, что для расстояний зондирования до $100 \mathrm{~m}$ и времени измерения $10 \mathrm{~s}$ диапазон изменения концентраций составляет около трех порядков от $10^{13}$ до $10^{17} \mathrm{~cm}^{-3}$ для всего диапазона расстояний зондирования $z$ до $100 \mathrm{~m}$.

Как следует из полученных результатов, для измерения концентрации молекул водорода $N(z)=10^{13} \mathrm{~cm}^{-3}$ в диапазоне зондирования от 5 до $100 \mathrm{~m}$ время измерения $t$ лежит в диапазоне от $3.83 \mathrm{~s}$ до $26.5 \mathrm{~min}$, для измерения концентрации $N(z)=10^{15} \mathrm{~cm}^{-3}$ - от $38 \mathrm{~ms}$ до $15.9 \mathrm{~s}$ и для измерения концентрации $N(z)=10^{17} \mathrm{~cm}^{-3}-$ от $0.4 \mu$ s до $160 \mu \mathrm{s}$.

Это значит, что в зависимости от ожидаемой реальной концентрации на заданном расстоянии зондирования можно будет по времени измерения выбрать параметры лазера, телескопа и фотоприемника для создания требуемой лидарной системы комбинационного рассеяния света молекулами водорода. Такой выбор определяется спектральной зависимостью дифференциальных сечений полосы колебательного комбинационного рассеяния света молекулами водорода, коэффициентов ослабления в атмосфере на этих длинах волн и относительной спектральной чувствительности фотоприемника.

Всюду в расчетах частота следования лазерных импульсов составляла $100 \mathrm{kHz}$ и время одного измерения $50 \mathrm{~ns}$ или шага по расстоянию $\Delta z=7.5 \mathrm{~m}$. Очевидно, что дальнейшее уменьшение концентрации исследуемых молекул возможно за счет увеличения частоты следования лазерных импульсов $f$ или увеличения их энергии $E_{0}$.

\section{Заключение}

Таким образом, полученные результаты позволяют выбрать оптимальные параметры лазера и лидара комбинационного рассеяния света для зондирования молекул водорода в атмосфере на расстояниях зондирования до $100 \mathrm{~m}$. Причем они хорошо согласуются с результатами наших прежних работ [4]. Чтобы зондировать молекулы водорода с концентрациями $N(z)$ от $10^{13}$ до $10^{17} \mathrm{~cm}^{-3}$ в диапазоне зондирования от 5 до $100 \mathrm{~m}$, достаточно времени измерения от $400 \mathrm{~ns}$ до $26.5 \mathrm{~min}$, что возможно реализовать в одном лидаре комбинационного рассеяния света в конкретных экспериментальных условиях $[3,4]$. Поэтому, подбирая время измерения за счет параметров того же варианта лидара, можно увеличить расстояние зондирования необходимых концентраций молекул водорода в атмосфере.

\section{Финансирование работы}

Работа выполнена при частичной финансовой поддержке грантов РФФИ, проекты № 19-42-230004 и № 19-45-230009.

\section{Конфликт интересов}

Авторы заявляют, что у них нет конфликта интересов.

\section{Список литературы}

[1] С.И. Козлов. Водородная энергетика: современное состояние, проблемы, перспективы (Газпром ВНИИГАЗ, M., 2009).

[2] Р. Межерис. Лазерное дистанционное зондирование (Мир, М., 1987).

[3] В.Е. Привалов, А.Э. Фотиади, В.Г. Шеманин. Лазеры и экологический мониторинг атмосферы (Лань, СПб., 2013).

[4] В.Е. Привалов, В.Б. Смирнов, В.Г. Шеманин. Расчет naраметров лазерного зондирования молекулярного водорода. (Препринт НИИ Российский центр лазерной физики, СПб., 1998).

[5] V.E. Privalov, V.G. Shemanin. Bulletin Russian Academy Sciences. Physics, 79 (2), 149 (2015).

[6] Г.Н. Глазов. Статистические вопросы лидарного зондирования атмосферы (Наука, Новосибирск, 1987).

[7] В.А. Донченко, М.В. Кабанов, Б.В. Кауль, И.В. Самохвалов. Атмосферная электрооптика (Изд. НТЛ, Томск, 2010).

[8] Лазерный контроль атмосферы, под. ред. Э.Д. Хинкли (Мир, М., 1979).

[9] Справочник по лазерам, под ред. А.М. Прохорова (Советское Радио, М., 1978), Т. I. 